\title{
NONLINEAR SPECTRAL IMAGING OF ELASTIC CARTILAGE IN RABBIT EARS
}

\author{
JING CHEN*, CHUNGEN GUO ${ }^{\dagger}$, HONGSHENG LI*, XIAOQIN ZHU**, \\ SHUYUAN XIONG ${ }^{\dagger}$ and JIANXIN CHEN*,§ \\ *Institute of Laser and Optoelectronics Technology \\ Fujian Provincial Key Laboratory for Photonics Technology \\ Key Laboratory of OptoElectronic Science and Technology for \\ Medicine of Ministry of Education \\ Fujian Normal University, Fuzhou 35000\%, P. R. China \\ ${ }^{\dagger}$ Department of Plastic Surgery \\ The First Affiliated Hospital of Fujian Medical University \\ Fujian Medical University, Fuzhou 350005, P. R. China \\ tzhuxq@fjnu.edu.cn \\ ${ }^{\S}$ chenjianxin@fjnu.edu.cn
}

Received 29 May 2013

Accepted 17 June 2013

Published 25 July 2013

\begin{abstract}
Elastic cartilage in the rabbit external ear is an important animal model with attractive potential value for researching the physiological and pathological states of cartilages especially during wound healing. In this work, nonlinear optical microscopy based on two-photon excited fluorescence and second harmonic generation were employed for imaging and quantifying the intact elastic cartilage. The morphology and distribution of main components in elastic cartilage including cartilage cells, collagen and elastic fibers were clearly observed from the high-resolution two-dimensional nonlinear optical images. The areas of cell nuclei, a parameter related to the pathological changes of normal or abnormal elastic cartilage, can be easily quantified. Moreover, the three-dimensional structure of chondrocytes and matrix were displayed by constructing three-dimensional image of cartilage tissue. At last, the emission spectra from cartilage were obtained and analyzed. We found that the different ratio of collagen over elastic fibers can be used to locate the observed position in the elastic cartilage. The redox ratio based on the ratio of nicotinamide adenine dinucleotide $(\mathrm{NADH})$ over flavin adenine dinucleotide (FAD) fluorescence can also be calculated to analyze the metabolic state of chondrocytes in different regions. Our results demonstrated that this technique has the potential to provide more accurate and comprehensive information for the physiological states of elastic cartilage.
\end{abstract}

Keywords: Multiphoton microscopy; two-photon excited fluorescence; second harmonic generation; spectral analysis; elastic cartilage.

This is an Open Access article published by World Scientific Publishing Company. It is distributed under the terms of the Creative Commons Attribution 3.0 (CC-BY) License. Further distribution of this work is permitted, provided the original work is properly cited. 


\section{Introduction}

Elastic cartilage is present in the ear pinna, external auditory meatus, eustachian tubes and epiglottis, etc. The major components of elastic cartilage are specialized cells called chondroblasts that produce a large amount of extracellular matrix (ECM) composed of Type II collagen fibers, abundant ground substance rich in proteoglycan and elastic fibers. ${ }^{1,2}$ The regulation of chondrocyte function by the ECM is critical to understanding normal physiological state of cartilage and its changes in disease states. Cartilage diseases include a wide variety of clinical phenotypes from common osteoarthrosis to several different types of chondrodysplasias. ${ }^{3,4}$ It is important to treat elastic cartilage defects or other cartilage diseases for improving the quality of life in patients. ${ }^{5}$ Although some therapies such as reconstruction surgery that depend on development in tissue engineering technique have been developed for repairing cartilage lesions, visualizing these complex processes within cartilage tissues remains challenging. ${ }^{6}$

Recently, nonlinear optical microscopy (NLOM) based on two-photon excited fluorescence (TPEF) and second harmonic generation (SHG) has become a powerful tool to observe unstained samples. ${ }^{7-10} \mathrm{It}$ presents a potential alternative for high resolution, minimally invasive in vivo imaging for early detection and serial monitoring of response to therapy in patients with cartilage disease. NLOM may utilize the natural intrinsic fluorescence properties of tissue to generate images that would allow visualization of the structural and cellular characteristics of elastic cartilage in fresh, unfixed tissue specimens. We therefore studied the arrangement and morphology of the chondrocytes and the surrounding matrix by using NOLM, in the elastic cartilage of the rabbit external ears.

\section{Materials and Methods}

\subsection{Sample preparations}

Three elastic cartilage tissues excised from experimental rabbits were examined in this study, provided by the Animal Center of Fujian Medical University. They were snap-frozen in liquid nitrogen (the temperature is $-196^{\circ} \mathrm{C}$ ) immediately to store before use. The tissue sections for imaging were cut transversely on a freezing microtome into $100 \mu \mathrm{m}$ thickness and sandwiched between the microscope slide and cover glass. The sections were imaged with the cover glass facing the microscopic objective. Moreover, a little PBS solution was dripped into the tissue specimen to avoid dehydration or shrinkage during the imaging process.

\subsection{NLOM system}

The nonlinear microscopic system used in this study contains a high-throughput scanning inverted Axiovert 200 microscope (LSM 510 META, Zeiss, Germany) equipped with a mode-locked femtosecond Ti:sapphire laser $(110 \mathrm{fs}, 76 \mathrm{MHz})$, tunable from 700 to $980 \mathrm{~nm}$ (Mira 900-F; Coherent, America). ${ }^{11,12}$ An objective (Plan-Apochromat 40X, N.A.1.4, Zeiss) was employed for focusing the excitation beam and for collecting the backward signals. The signals were directed by a main dichroic beam splitter (MDBS) to the META detector. The system has two imaging modes: channel mode and lambda mode. The channel mode can achieve SHG and TPEF imaging, while the lambda mode can carry out spectral imaging and obtain emission spectra of regions of interest (ROI) within the spectral image. In this work, two different channels of the META detector were selected to collect the backward SHG and TPEF signals. One channel is corresponding to the wavelength range of $398-409 \mathrm{~nm}$ to show microstructure of collagen SHG signals when the excitation wavelength $\lambda_{\text {ex }}=810 \mathrm{~nm}$ were used, whereas the other one covered the wavelength range of $430-697 \mathrm{~nm}$ in order to image TPEF signals. Using the image-guided spectral analysis method, the intensity spectrum of emission signal from elastic cartilage was recorded and analyzed. The images were obtained at $2.5 \mu$ s per pixel. All images had a 12-bit pixel depth.

All data were presented as a mean value with its standard deviation indicated (mean \pm SD) and analyzed using Excel software.

\section{Results and Discussion}

\subsection{High-resolution NLOM image of intact elastic cartilage}

An intact elastic cartilage is composed of large chondrocytes surrounded by an extensive ECM (including mainly collagen and elastic fibers) and covered with perichondrium. Morphological features of elastic cartilage in the rabbit external ear can be 


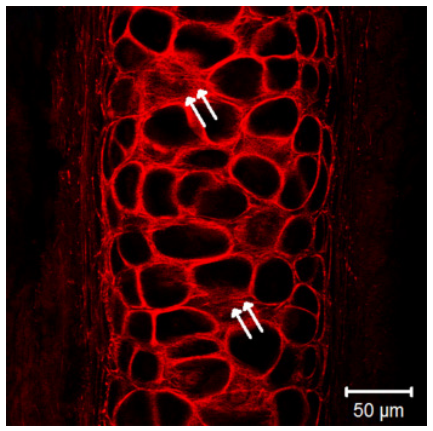

(a)

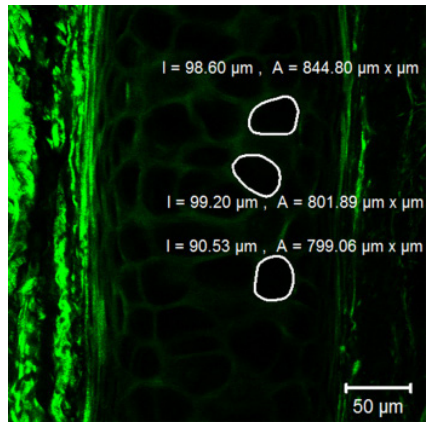

(b)

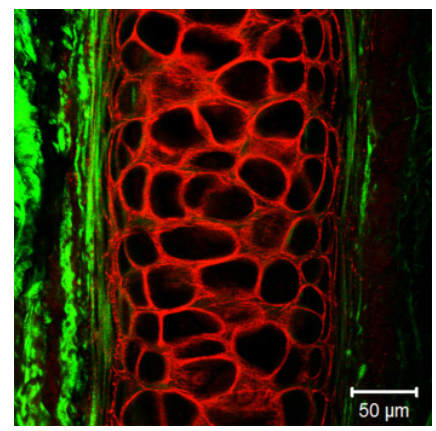

(c)

Fig. 1. Representative NLOM images of elastic cartilage in the rabbit external ear. (a) TPEF (red, 430 to $697 \mathrm{~nm}$ ), (b) SHG (green, 398 to $409 \mathrm{~nm}$ ), and (c) TPEF/SHG merged obtained by overlaying two channels. It clearly shows the elastic fibers (white arrows) and chondrocytes (white round-enclosed regions). The excited wavelength $\lambda_{\text {ex }}=810 \mathrm{~nm}$. Scale bar $=50 \mu \mathrm{m}$.

well resolved by TPEF/SHG images. Representative 2D TPEF/SHG images of an intact elastic cartilage for an excitation wavelength $\lambda_{\mathrm{ex}}=810 \mathrm{~nm}$ were shown in Fig. 1. The collagen fibers surrounding the chondrocytes in elastic cartilage were well revealed by SHG signals in green color-code, shown in Fig. 1(b). The elastic fibers (white arrows) and intracellular compounds were effective for generating TPEF signals in red color-code, shown in Fig. 1(a). The chondrocytes can be identified and show the nonfluorescent nuclei displayed darkly on the optical section surrounded by ECM, and the clear cellular boundary was shown. Combined TPEF and SHG image was shown in Fig. 1(c), displaying the distinct elastic cartilage morphology and providing complementary information on the chondrocytes, perichondrium and ECM. It can be seen that fully differentiated chondrocytes in the middle portion of the cartilage were round, while immature cells near the periphery were oblong with their long axes parallel to the perichondrium. The perimeters and areas of the cells can be directly measured by using system analyzing tools, seen by the white round-enclosed regions in Fig. 1(b). The average of the perimeters and areas of the cells were $93.98 \pm 4.84 \mu \mathrm{m}$ and $789.08 \pm 25.63 \mu \mathrm{m}^{2}$, respectively. The changes in cell size may be an indicator for evaluating pathological changes of normal or abnormal elastic cartilage. In addition, the ECM is an "information rich" environment and interactions between the chondrocytes. The density of ECM can also be quantified and used to assess pathological processes.

Moreover, 10 sequential SHG/TPEF overlapping images at intervals of $2 \mu \mathrm{m}$ were given in Fig. 2,
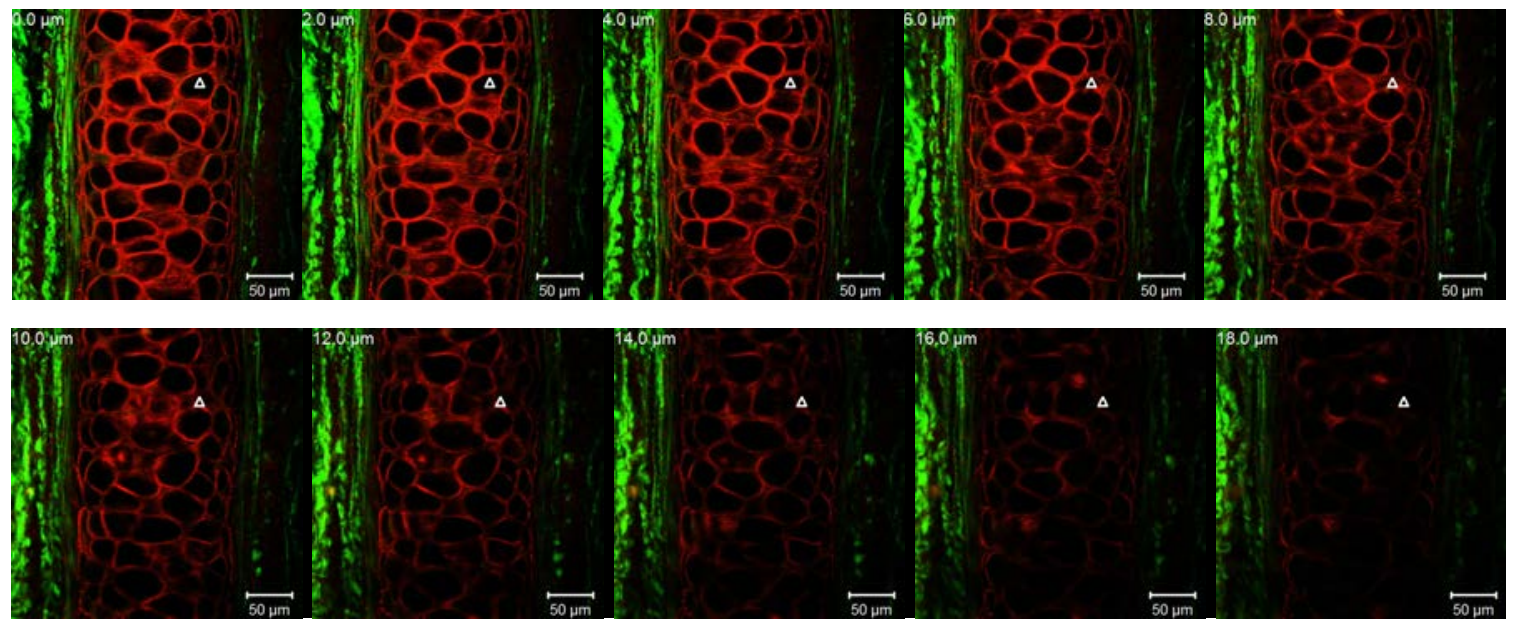

Fig. 2. A total of 10 sequential SHG/TPEF overlapping images at intervals of $2 \mu \mathrm{m}$, providing the 3D shape of chondrocytes and the morphology of the surrounding collagen and elastic fibers. The triangle points to a chondrocyte to visualize the shape varied with depth. Scale bar $=50 \mu \mathrm{m}$. 


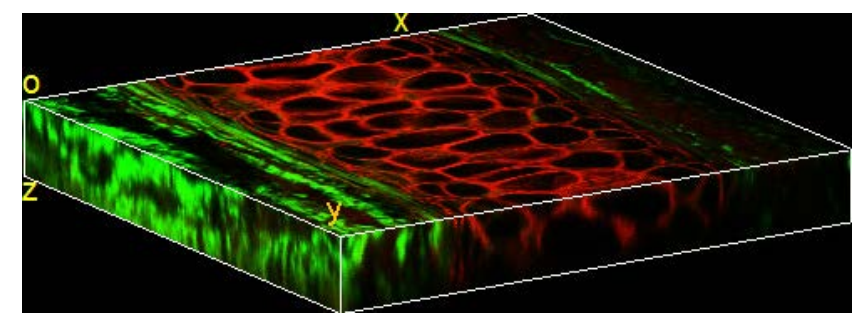

Fig. 3. 3D reconstruction of the sequential images shown in Fig. 2.

proving the ultrastructure of chondrocytes and the surrounding matrix proteins varied with imaging depth. The $3 \mathrm{D}$ reconstruction of the sequential images was given by Fig. 3, more distinctly showing the 3D shape of chondrocytes and morphological structures of ECM in elastic cartilage.

\subsection{Depth-resolved spectral imaging and emission spectra of elastic cartilage}

To determine the precise origin of image-forming signals from elastic cartilage, the emission spectra from the elastic cartilage regions were acquired using the image-guide spectral analysis method for $\lambda_{\text {ex }}=810 \mathrm{~nm}$. Figure 4(a) presented the spectral resolved image. Figure 4(b) displayed representative spectra measured from the chondrocytes and ECM at the center of elastic cartilage region (red rectangle-enclosed region in Fig. 4(a), ROI 1), the peripheral region of the cartilage (green rectangleenclosed region in Fig. 4(a), ROI 2), and the perichondrium region (blue rectangle-enclosed region in Fig. 4(a), ROI 3). Each spectrum revealed a peak at
$405 \mathrm{~nm}$ (exactly half the excitation wavelength) and a broad spectral feature characteristic of TPEF. The peak at $405 \mathrm{~nm}$ has a quadratic dependence on incident laser intensity and shifts with changes in laser frequency to remain at exactly half the excitation wavelength. ${ }^{9}$ Fluorescence spectra contained multiple overlapping peaks, especially including three peaks of interest centered at $\sim 500, \sim 475$ and $\sim 535 \mathrm{~nm}$ resulting from elastin, $\mathrm{NADH}$ and FAD in cells, respectively. ${ }^{13,14}$ It showed different signal intensities for various ROIs. In ROI 1, the SHG signal intensity was obviously lower than the TPEF signal. In ROI 2, the SHG signal intensity was comparable to the TPEF signal. And the ROI 3 exhibited a stronger SHG signal than the TPEF signal due to the condensed collagen fibers in the perichondrium region.

The peak intensities of collagen SHG $(\sim 405)$ and elastin TPEF $(\sim 500)$ signals were defined as A and $\mathrm{B}$, respectively. Then the ratio of collagen over elastin was calculated by using the following formula: A/B. As a result, the ratios of the three regions were $0.62 \pm 0.04$ for ROI $1,1.23 \pm 0.09$ for ROI 2 and $9.65 \pm 0.42$ for ROI 3 , showing significant difference.

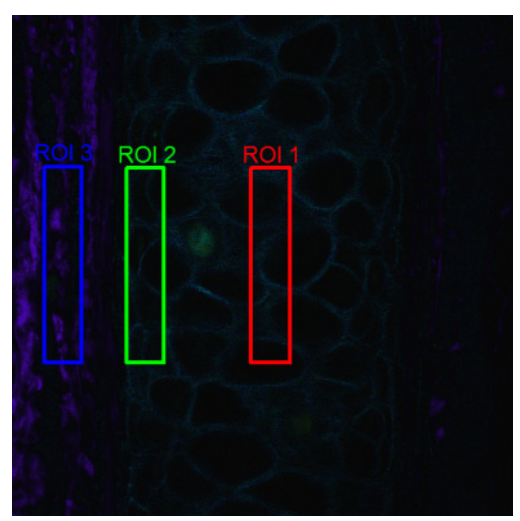

(a)

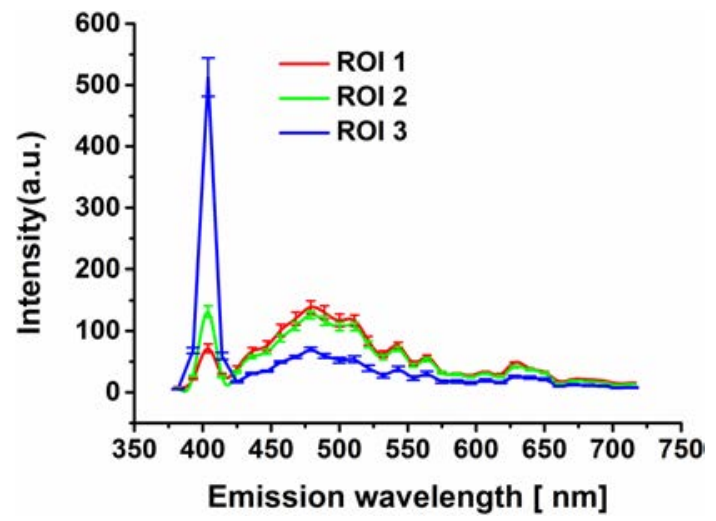

(b)

Fig. 4. (a) Spectral resolved TPEF/SHG image and (b) emission spectra obtained from the center region (red rectangle-enclosed region, ROI 1), peripheral region (green rectangle-enclosed region, ROI 2), and the perichondrium region (blue rectangle-enclosed region, ROI 3) of elastic cartilage. The excited wavelength $\lambda_{\mathrm{ex}}=810 \mathrm{~nm}$. 
It indicated that the ratio of collagen over elastic fibers can be used for locating the position observed in the cartilage. In addition, it has been reported that the ratio of NADH over FAD fluorescence calculated by the equation, $\mathrm{FAD}_{535} /\left(\mathrm{FAD}_{535}+\mathrm{NADH}_{475}\right)$, is a parameter related to cellular metabolism. ${ }^{15,16}$ In this study, the ratio of NADH over FAD fluorescence in ROI 1 region was $1.81 \pm 0.03$, which reflected the metabolic state of chondrocytes and may be found application in characterization of pathological changes for distinguishing abnormal elastic cartilage from an intact one.

\section{Conclusion}

In conclusion, NLOM has been demonstrated to be an effective technique to image fine structure of elastic cartilage in the rabbit external ear, without the need for additional fluorescent probe. We demonstrated that the technique can provide highcontrast visualization of elastic cartilage components, and identify cellular, perichondrium and ECM. Coupled with several system analyzing tools, the technique allowed analysis of intrinsic spectra in tissue, quantitatively providing some important information on the biomorphology and biochemistry of the elastic cartilage. With the advance of this technique, NLOM can provide the accurate and comprehensive information for the clinical research of elastic cartilage tissues.

\section{Acknowledgments}

The project was supported by the Program for Changjiang Scholars and Innovative Research Team in University (Grant No. IRT1115) and the National Natural Science Foundation of China (Grant Nos. 81101209, 61275006, 81271620).

\section{References}

1. A. T. Yeh, M. J. Hammer-Wilson, D. C. Van Sickle, "Nonlinear optical microscopy of articular cartilage," Osteoarthr. Cartil. 13, 345-352 (2005).

2. E. H. Nielsen, P. Bytzer, "High resolution scanning electron microscopy of elastic cartilage," J. Anat. 129, 823-831 (1979).

3. M. Vikkula, M. Metsaranta, L. A. Kokko, "Type II collagen mutations in rare and common cartilage diseases," Ann. Med. 26, 107-114 (1994).

4. R. F. Loeser, "Chondrocyte integrin expression and function," Biorheology 37, 109-116 (2000).
5. K. Ulutas, A. Menderesa, C. Karacaa, S. Ozkalb, "Repair of cartilage defects with periosteal grafts," Br. J. Plast. Surg. 58, 65-72 (2005).

6. B. H. I. Ruszymah, B. S. Lokman, A. Asma, S. Munirah, K. Chua, A. L. Mazlyzam, M. R. Isa, N. H. Fuzina, B. S. Aminuddin, "Pediatric auricular chondrocytes gene expression analysis in monolayer culture and engineered elastic cartilage," Int. J. Pediatr. Otorhinolaryngol. 71, 1225-1234 (2007).

7. W. R. Zipfel, R. M. Williams, R. Christie, A. Y. Nikitin, B. T. Hyman, W. W. Webb, "Live tissue intrinsic emission microscopy using multiphotonexcited native fluorescence and second harmonic generation," Proc. Natl. Acad. Sci. USA 100, 7075-7080 (2003).

8. J. L. Qu, L. X. Liu, Y. H. Shao, H. B. Niu, B. Z. Gao, "Recent progress in multifocal multiphoton microscopy," J. Innov. Opt. Health Sci. 05, 1250018 (2012).

9. R. A. Xu, X. Q. Zhu, N. He, S. M. Zhuo, J. Xu, S. H. Jiang, H. S. Li, J. X. Chen, "Multiphoton Microscopic imaging of mouse intestinal mucosa based on two-photon excited fluorescence and second harmonic generation," J. Innov. Opt. Health Sci. 06, 1350004 (2013).

10. L. Silvestri, A. L. Allegra Mascaro, J. Lotti, L. Sacconi, F. S. Pavone, "Advanced optical techniques to explore brain structure and function," $J$. Innov. Opt. Health Sci. 06, 1230002 (2013).

11. X. Q. Zhu, S. M. Zhuo, L. Q. Zheng, X. S. Jiang, J. X. Chen, B. F. Lin, "Characteristics of scar margin dynamic with time based on multiphoton microscopy," Lasers. Med. Sci. 26, 239-245 (2011).

12. X. S. Jiang, S. M. Zhuo, J. X. Chen, "Diagnostic application of multiphoton microscopy in epithelial tissues," J. Innov. Opt. Health Sci. 04, 159-163 (2011).

13. J. X. Chen, S. M. Zhuo, R. Chen, X. S. Jiang, S. S. Xie, Q. L. Zou, "Depth-resolved spectral imaging of rabbit esophageal tissue based on two-photon excited fluorescence and second-harmonic generation," New J. Phys. 9, 212 (2007).

14. H. C. Bao, A. Boussioutas, M. Aleixandria, R. Busuttil, M. Gu, "Gastrointestinal abnormalities identified by fluorescence endomicroscopy," J. Innov. Opt. Health Sci. 05, 1250026 (2012).

15. S. M. Zhuo, J. X. Chen, B. Y. Yu, X. S. Jiang, T. Luo, Q. G. Liu, R. Chen, S. S. Xie, "Nonlinear optical microscopy of the bronchus," J. Biomed. Opt. 13, 0540240 (2008).

16. S. Huang, A. A. Heikal, W. W. Webb, "Two-photon fluorescence spectroscopy and microscopy of NAD $(\mathrm{P}) \mathrm{H}$ and flavoprotein," Biophys. J. 82, 2811-2825 (2002). 